Marquette University

e-Publications@Marquette

Mechanical Engineering Faculty Research and

Publications

Mechanical Engineering, Department of

9-14-2003

\title{
Sufficient Conditions for Admittance to Ensure Planar Force-assembly in Multi-point Frictionless Contact
}

Shuguang Huang

Marquette University, shuguang.huang@marquette.edu

Joseph M. Schimmels

Marquette University, joseph.schimmels@marquette.edu

Accepted version. Published as a part of the Proceedings. ICRA '03. IEEE International Conference on Robotics and Automation, 2003. DOI. (C) 2003 IEEE. Used with permission. 


\section{Marquette University \\ e-Publications@Marquette}

\section{Mechanical Engineering Faculty Research and Publications/College of Engineering}

This paper is NOT THE PUBLISHED VERSION; but the author's final, peer-reviewed manuscript. The published version may be accessed by following the link in the citation below.

IEEE Transactions on Robotics and Automation, Vol. 3 (2003): 3095-3100. DOI. This article is (C) Institute of Electrical and Electronics Engineers and permission has been granted for this version to appear in e-Publications@Marquette. IEEE does not grant permission for this article to be further copied/distributed or hosted elsewhere without the express permission from IEEE.

\section{Sufficient Conditions for Admittance to Ensure Planar Force-Assembly in Multi- Point Frictionless Contact}

Shuguang Huang

Dept. of Mech. \& Ind. Eng., Marquette University, Milwaukee, WI

J.M. Schimmels

Dept. of Mech. \& Ind. Eng., Marquette University, Milwaukee, WI

\section{SECTION I.}

\section{Introduction}

Admittance control has been used in assembly tasks to provide force regulation and force guidance. In these tasks, the admittance maps contact forces into changes in the velocity of the held body. To achieve reliable assembly through force-guidance, an appropriate 
admittance must be selected. For linear admittance behavior, the planar control law has the form:

$$
\mathbf{v}=\mathbf{v}_{0}+\mathbf{A w} \text { (1) }
$$

where $v_{0}$ is the nominal velocity (a 3-vector), $\mathbf{w}$ is the contact wrench measured in the body frame (a 3-vector), and $\mathbf{A}$ is the admittance matrix (a $3 \times 3$ matrix).

Many researchers have addressed the use of an admittance for force-guidance. Whitney [1] [2] proposed that the compliance of a manipulator be structured so that contact forces lead to decreasing errors. Peshkin ${ }^{[3]}$ addressed the synthesis of an accommodation (inverse damping) matrix by specifying the desired force/motion relation at a sampled set of positional errors for a planar assembly task. An unconstrained optimization was then used to obtain an accommodation matrix. Asada ${ }^{[4]}$ used a similar optimization procedure for the design of an accommodation neural network rather than an accommodation matrix. Others ${ }^{[5]}{ }^{[6]}$ provided synthesis procedures based on spatial intuitive reasoning. None of these approaches, however, ensures that the admittance selected will, in fact, be reliable.

A reliable admittance selection approach is to design the control law so that, at each possible part misalignment, the contact force always leads to a motion that reduces the existing misalignment. The approach is referred to as force-assembly [7], [8], [9]. A condition for forceassembly is that the admittance matrix $\mathbf{A}$ is positive semidefinite ${ }^{[7]}$.

For force-assembly, the motion resulting from contact must instantaneously reduce misalignment. Since the configuration space of a rigid body is non-Euclidian, there is no natural metric for finite error. In ${ }^{[10]}$, several body-specific rigid body metrics were identified. These metrics are based on the Euclidean distance between one (or more) point on the body and its corresponding location when properly positioned.

Previously, we have considered sufficient conditions on the admittance to ensure planar forceassembly in frictionless single-point contact [11], [12]. In the study, we considered a measure of error based on the Euclidean distance between a single (fixed) point on the held body and its location when properly positioned. The error reduction conditions developed require that, at each possible misalignment, the contact force yields a motion that reduces this distance. A set of sufficient conditions was identified that ensures planar force-assembly in single-point contact.

This paper considers planar rigid body assembly in multi-point frictionless contact. The mathematical description of error-reducing motion for two-point contact is derived in Section II. The solution strategy is presented in Section III. The strategy ensures that conditions imposed at a finite number of contact configurations guarantee that the conditions are also satisfied at the infinite number of contact configurations within a specified range. Sections IV addresses the sufficient conditions for each of the various types of multi-point contact. A brief summary is presented in Sections V. 


\section{SECTION II.}

\section{Error-Reducing Motion}

In this section, the error-reducing motion of a constrained rigid body in two-point contact is analyzed. First, the equation describing the constrained motion of a rigid body is derived. Then, the error-reduction function for general two-point contact is obtained.

\section{A. Constrained Rigid Body Motion}

Consider planar motion of a rigid body in 2-point contact with another part. Let $\mathbf{n}_{i}$ be the surface normal at contact point $i$ and let $\phi_{i} \geq 0$ be the magnitude of the normal force. Then, for two-point contact, the total contact wrench is

$$
\mathbf{w}=\mathbf{w}_{1} \phi_{1}+\mathbf{w}_{2} \phi_{2}(2)
$$

where

$$
\mathbf{w}_{i}=\left[\begin{array}{c}
\mathbf{n}_{i} \\
\left(\mathbf{r}_{i} \times \mathbf{n}_{i}\right) \cdot \mathbf{k}
\end{array}\right]
$$

and where $\mathbf{r}_{i}$ is the position vector from the origin of the coordinate frame to the contact point and $\mathrm{k}$ is the unit vector orthogonal to the plane.

Alternately, if we denote

$$
\mathbf{W}=\left[\mathbf{w}_{1}, \mathbf{w}_{2}\right] \in \mathbb{R}^{3 \times 2}, \phi=\left[\phi_{1}, \phi_{2}\right]^{T} \in \mathbb{R}^{2},
$$

then the total contact wrench is: $\mathbf{w}=\mathbf{W} \phi$

By the control law (1), the motion of the rigid body is given by:

$$
\mathbf{v}=\mathbf{v}_{0}+\mathbf{A W} \phi \cdot(3)
$$

To maintain contact ${ }^{[13]}$, the reciprocal condition requires:

$$
\mathbf{w}_{i}^{\mathrm{T}} \mathbf{v}=0 \Rightarrow \mathbf{W}^{T} \mathbf{v}=0 .
$$

Substituting this into (3) and solving for $\phi$, we have:

$$
\phi=-\left[\mathbf{W}^{T} \mathbf{A} \mathbf{W}\right]^{-1} \mathbf{W}^{T} \mathbf{v}_{0} \cdot(4)
$$

Thus the equation for constrained motion is obtained: 


$$
\mathbf{v}=\mathbf{v}_{0}-\mathbf{A W}\left[\mathbf{W}^{T} \mathbf{A} \mathbf{W}\right]^{-1} \mathbf{W}^{T} \mathbf{v}_{0} \cdot(5)
$$

\section{B. Error-Reduction Function}

For planar motion of a rigid body with two point contact, if the contact is maintained, the body has only one degree of freedom (DOF). The instantaneous motion of the body is a rotation about the body's instantaneous center.

If the instantaneous center is at infinity, the motion of the body is a pure translation. This is the simplest case: the error-reduction at any given configuration within a contact state ensures the error-reduction for all configurations within that contact state.

If the instantaneous center is not at infinity (generic case), it is uniquely determined by the geometry of the contact for each configuration. This paper addresses the contact states of this case.

As stated previously, assembly error-reduction requires that, at any instant, the motion of the body must be toward its properly mated position. Consider the two-point contact state shown in Fig. 1. For error-reduction, the direction of rotation of the body about the instantaneous center ci must cause the body to move toward the properly mated position $B^{\prime}$. Since error-reduction must hold for any configuration, the angular motion of the body must be along a specific direction for all configurations within the same contact state. Thus, the error-reducing motion for two-point contact is solely indicated by the angular velocity of the constrained body.

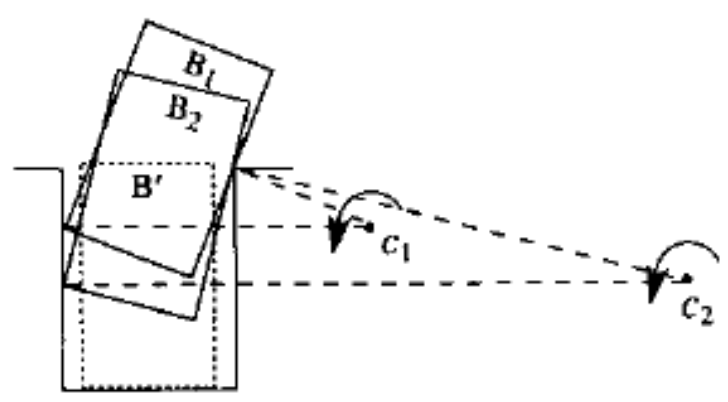

Fig. 1. Error-Reducing Motion: the angular motion of the rigid body must be along a specific direction for all configurations of a given two-point contact state.

Now consider the angular motion in (5). Let $\mathbf{e}_{3}=[0,0,1]^{T}$ and $\mathbf{v}_{0}=\left[v_{x 0}, v_{y 0}, \omega_{0}\right]^{T}$, then, the orientational component in (5) is:

$$
\omega=\mathbf{e}_{3}^{T} \mathbf{v}=\omega_{0}-\mathbf{e}_{3}^{\mathbf{T}} \mathbf{A} \mathbf{W}\left[\mathbf{W}^{\mathbf{T}} \mathbf{A} \mathbf{W}\right]^{-1} \mathbf{W}^{T} \mathbf{v}_{0} \cdot(6)
$$

Let $\left[\mathbf{W}^{\mathrm{T}} \mathbf{A W}\right]^{*}$ be the adjugate of $\left[\mathbf{W}^{T} \mathbf{A W}\right]$. Then $(6)$ can be written as:

$$
\omega=\frac{\operatorname{det}\left(\mathbf{W}^{T} \mathbf{A W}\right) \omega_{0}-\mathbf{e}_{3}^{T} \mathbf{A W}\left[\mathbf{W}^{T} \mathbf{A W}\right]^{*} \mathbf{W}^{T} \mathbf{v}_{0}}{\operatorname{det}\left(\mathbf{W}^{T} \mathbf{A} \mathbf{W}\right)} \text {. (7) }
$$


Since $\mathrm{A}$ is positive definite, $\operatorname{det}\left(\mathbf{W}^{\mathrm{T}} \mathbf{A W}\right)>0$ and we only need to consider the following function:

$$
F_{e r}=\operatorname{det}\left(\mathbf{W}^{T} \mathbf{A W}\right) \omega_{0}-\mathbf{e}_{3}^{T} \mathbf{A} \mathbf{W}\left[\mathbf{W}^{T} \mathbf{A} \mathbf{W}\right]^{*} \mathbf{W}^{T} \mathbf{v}_{0}
$$

which can be expressed as:

$$
\begin{aligned}
F_{e r}=\left[\left(\mathbf{w}_{1}^{T}\right.\right. & \left.\left.\mathbf{A} \mathbf{w}_{1}\right)\left(\mathbf{w}_{2}^{T} \mathbf{A} \mathbf{w}_{2}\right)-\left(\mathbf{w}_{1}^{T} \mathbf{A} \mathbf{w}_{2}\right)^{2}\right] \omega_{0} \\
& -\left(\mathbf{w}_{1}^{T} \mathbf{v}_{0}\right)\left(\mathbf{a}_{3}^{T} \mathbf{w}_{1}\right)\left(\mathbf{w}_{2}^{T} \mathbf{A} \mathbf{w}_{2}\right) \\
& +\left(\mathbf{w}_{1}^{T} \mathbf{v}_{0}\right)\left(\mathbf{a}_{3}^{T} \mathbf{w}_{2}\right)\left(\mathbf{w}_{1}^{T} \mathbf{A} \mathbf{w}_{2}\right) \\
& +\left(\mathbf{w}_{2}^{T} \mathbf{v}_{0}\right)\left(\mathbf{a}_{3}^{T} \mathbf{w}_{1}\right)\left(\mathbf{w}_{1}^{T} \mathbf{A} \mathbf{w}_{2}\right) \\
& -\left(\mathbf{w}_{2}^{T} \mathbf{v}_{0}\right)\left(\mathbf{a}_{3}^{T} \mathbf{w}_{2}\right)\left(\mathbf{w}_{1}^{T} \mathbf{A} \mathbf{w}_{1}\right)
\end{aligned}
$$

where $\mathbf{a}_{3}$ is the $3 r d$ column of the admittance matrix $A$.

Since the function $F_{e r}$ in (8) indicates the sign of the orientational motion for the body, it is used as the error-reduction function for the two-point contact case. If, error-reducing motion is achieved at one configuration and the angular velocity of the body (indicated by Fer) does not change sign within the range of configurations, then error-reduction is ensured for all configurations within the contact state.

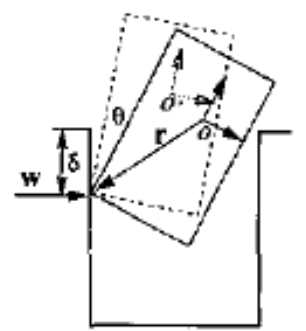

(a)

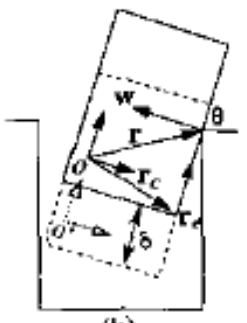

(b)

Fig. 2. Single-point Contact State. (a) $\{v-e\}$ : vertex-edge contact state. (b) $\{e-v\}$ : edge-vertex contact state.

\section{SECTION III.}

\section{Solution Strategy}

In general, the error-reduction function Fer in (8) depends on the geometries of the parts in contact. In this section, a solution strategy to obtain sufficient conditions for error-reduction is presented. With this strategy, a set of sufficient conditions can be obtained for bounded configurations without having to explicitly describe the variation in part configuration within a given contact state. 


\section{A. Contact States}

Polygonal planar bodies in single-point contact have two basic types of contact. One is referred to as "vertex-edge" contact $(\{v-e\})$; the other is referred to as "edge-vertex" contact $(\{e-v\})$. In "vertex-edge" contact, one vertex of the held body is in contact with one edge of its mating part (Fig. 2a). In "edge-vertex" contact, one edge of the held body is in contact with one vertex of the mating fixtured part (Fig. 2b).

The basic types of two-point contact are the various combinations of two single-point contacts. There are three types of two-point contact: 1$)$ one $\{e-v\}$ and one $\{v-e\}$ contact $(\{e-v, v-e\})$ two $\{e-v)$ contact $(\{e-v, e-v\})$, and 3) two $\{v-e\}$ contact $(\{v-e, v-e\})$.

\section{B. Contact Wrenches}

In the following, the contact wrenches for the two basic types of single-point contact are presented. We show that, although the body configuration is determined by two variables $(\delta, \theta)$, the unit contact wrench for each type of contact only depends on one of them. As a consequence, two-point contact is readily expressed in terms of two variables.

\section{Vertex-Edge Contact}

Consider the case for which one vertex of the body is in contact with one edge of its mating part $(\{v-e\}$ contact state). As shown in Fig. $2 a$, the direction of the contact wrench $w$ is constant in the global coordinate frame and the relative position of the contact is constant in the body frame. Suppose that the relative body orientation is given by angle $\theta$, then the direction of the contact force also changes in $\theta$ in the body frame. Thus, the unit contact wrench in the body frame can be expressed as:

$$
\mathbf{w}=\left[\begin{array}{c}
\mathbf{R n} \\
(\mathbf{r} \times \mathbf{R n}) \cdot \mathbf{k}
\end{array}\right]
$$

where $\mathrm{n}$ is the surface normal for a chosen configuration and $\boldsymbol{R}$ is the rotation matrix associated with $\theta$ having the form:

$$
\mathbf{R}=\left[\begin{array}{cc}
\cos \theta & -\sin \theta \\
\sin \theta & \cos \theta
\end{array}\right] \cdot
$$

Therefore, in the body frame, w is a single-variable function in $\theta$ for a specified contact state.

\section{Edge-Vertex Contact}

Consider the case for which one edge of the body is in contact with one vertex of its mating part ( $\{e-v\}$ contact state). As shown in Fig. 2b, the direction of the contact wrench is constant in the body frame but the location of the contact varies, thus the unit contact wrench can be expressed as: 


$$
\mathbf{w}=\left[\begin{array}{c}
\mathbf{n} \\
\left.\left[\left(\mathbf{r}_{c}+\mathbf{r}_{e} \delta\right) \times \mathbf{n}\right] \cdot \mathbf{k}\right]
\end{array}\right.
$$

where $\mathbf{r}_{c}$ identifies a location on the edge and $\mathbf{r}_{e}$ is the unit vector along the edge (both are constant in the body frame). Thus, $\mathrm{w}$ is a single-variable function in $\delta$ for a given contact state.

\section{Multi-Point Contact}

Since two-point contact is a combination of the two single-point contact cases, the contact wrench for two-point contact is a combination of the two corresponding single-point contact wrenches.

The error-reduction function for two-point contact [calculated using (8)] involves the two contact wrenches $\mathbf{w}_{1}$ and $\mathbf{w}_{2}$. Since each unit wrench in [(9) or (11)] is a function of $\delta$ or $\theta$, in general, the error-reduction function can always be expressed as a function of two variables, i.e.,

$$
F_{e r}=F_{e r}(\xi, \eta)(12)
$$

where $\xi$ and $\eta$ are $\theta$ or $\delta$ depending on the contact state. For example, for $\{e-v, e-v\}$ contact, both $\xi$ and $\eta$ involve the two displacement variables along the corresponding edges, $\delta_{1}$ and $\delta_{2}$.

C. Mathematical Requirement

If the parts remain in contact, the planar motion of the rigid body has only $1 \mathrm{DOF}$. Therefore, the two parameters $\xi$ and $\eta$ in (12) must be related by the geometry of the parts. For example, for a given geometry, $\xi$ can be expressed as a function of $\eta$ (or vice versa). The function, however, could be complicated (highly nonlinear) for a specific geometry.

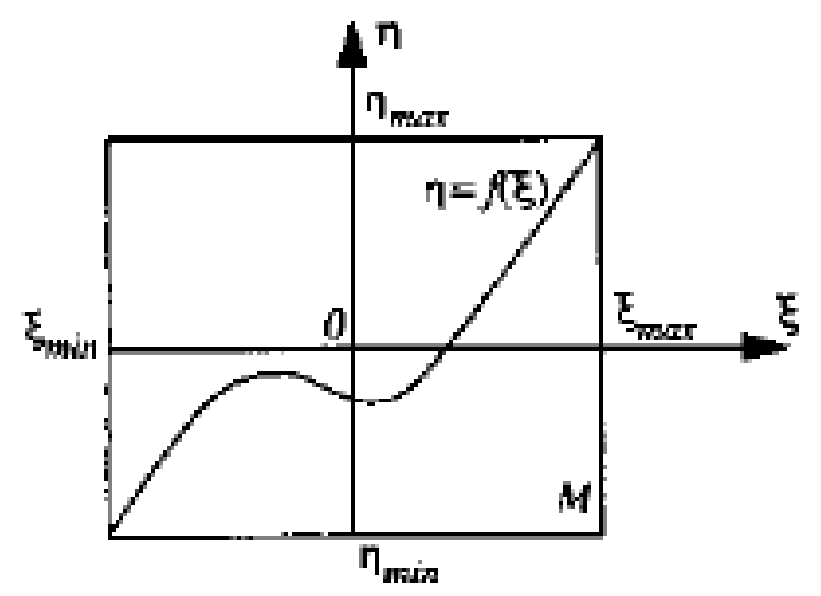

Fig. 3. Two-point Contact State: in the bounded area $\xi$ and $\eta$ are treated as two independent variables regardless of their relation $\eta=f(\xi)$. 
Note that $\delta$ represents a relative position along an edge of the held body and $\theta$ represents a relative orientation between the two parts. The ranges for $\delta$ and $\theta$ can be determined from bounds on relative misalignment or by bounds determined by the contact state. Thus the range of the two parameters $\xi$ and $\eta$ are readily determined.

For a given geometry and contact state, $\xi$ and $\eta$ are related by a function $\eta=f(\xi)$. The errorreduction condition requires that for all configurations on the curve $\eta=f(\xi)$, the orientational error of the body is monotonically reduced by contact. This means that the error-reduction function $F_{e r}$ has the appropriate sign along the curve $\eta=f(\xi)$.

Since the function is geometry specific and difficult to determine, we consider a set of more conservative conditions based on the range of the two variables.

Suppose that the range of $\xi$ and $\eta$ are $\left[\xi_{\min }, \xi_{\max }\right]$ and $\left[\eta_{\min }, \eta_{\max }\right]$, respectively. Consider the rectangular area $M$ bounded by $\left[\xi_{\min }, \xi_{\max }\right]$ and $\left[\eta_{\min }, \eta_{\max }\right]$ as shown in Fig. 3 . If in the bounded area the error-reduction condition is satisfied, then for any configuration considered, the error-reduction condition must be satisfied. Mathematically, this condition can be imposed on the function $F_{e r}$ as:

1. For one point $\left(\xi_{0}, \eta_{0}\right) \in M$, the error-reduction condition is satisfied, i.e.,

$$
F_{\epsilon r}=F_{e r}\left(\xi_{0}, \eta_{0}\right)<0
$$

1. For all points in $M, F_{e r}$ does not change sign.

As such, sufficient conditions for error-reduction motion are established. This approach enables us to treat the parameters $(\xi, \eta)$ as two independent variables regardless of the geometrical relationship between them.

For the variables $(\xi, \eta)$, Fer can be written as a polynomial in $\xi$ with the coefficients being functions of $\eta$. Since both variables $\xi$ and $\eta$ are bounded, the extremals of the coefficients can be determined. Based on these extremals a single-variable polynomial is constructed. Using an approach similar to that used for the single-point contact case [12], sufficient conditions for (13) are obtained. Since the conditions impose constrains on the admittance matrix, a set of sufficient conditions for an admittance to ensure force-assembly is identified.

\section{SECTION IV.}

\section{Conditions for Two-Point Contact}

In this section, the sufficient conditions are obtained for each type of contact state. Below, for each type of contact, 1) the error-reduction function $F_{e r}$ is specified, 2) bounds of the coefficients in $F_{e r}$ are identified, and 3) specific conditions for satisfying error-reduction are presented. 
In each case, the range for each of the variables can be transformed to be centered about zero, e.g., $\left[\delta_{\min }, \delta_{\max }\right] \Rightarrow\left[-\delta_{M}, \delta_{M}\right]$ to facilitate subsequent analysis.

A. Conditions for $\{e-v, v-e\}$ Contact

In this case, the two-point contact wrench is a combination of the two corresponding singlepoint contact wrenches.

Using the notation developed in Section III-B, the contact wrenches for the $[v-e]$ and $\{e-v\}$ contact are obtained by (9) and (11) respectively,

$$
\mathbf{w}_{1}=\mathbf{w}_{1}(\theta), \mathbf{w}_{2}=\mathbf{w}_{2}(\delta) \text {. }
$$

Since $\mathbf{w}_{1}$ in (9) contains only first order terms in $\cos \theta$ and $\sin \theta$, and $\mathbf{w}_{2}$ in (11) contains only a linear term in $\delta,(8)$ can be expressed as a function of $(\theta, \delta)$ in the form:

$$
F_{e r}(\theta, \delta)=q_{2} \delta^{2}+q_{1} \delta+q_{0}
$$

where $q_{i}$ 's are functions of $\theta$ having the forms:

$$
q_{i}=a_{i} \cos ^{2} \theta+b_{i} \cos \theta \sin \theta+c_{i} \cos \theta+d_{i} \sin \theta+e_{i}
$$

If at a given configuration error-reduction is satisfied: $F_{e r}<0$, then in order for all $\theta$ and $\delta$ to satisfy the condition, we need to obtain conditions such that $F_{e r}$ has no root in the range of consideration.

\section{A.1 Bounds on the Coefficients}

In order to analyze the root of the function $F_{e r}$, we evaluate the bounds on the coefficients $q_{i}$ 's in (16).

First, consider the two terms involving $\cos \theta$ alone $\left(a_{i} \cos ^{2} \theta+c_{i} \cos \theta\right)$ in (16). If we denote:

$$
\begin{gathered}
p_{i 1}^{+}=\max \quad\left\{\left(a_{i} \cos ^{2} \theta_{M}+c_{i}\right),\left(a_{i} \cos ^{2} \theta_{M}+c_{i} \cos \theta_{M}\right),\right. \\
\left.\left(a_{i}+c_{i} \cos \theta_{M}\right),\left(a_{i}+c_{i}\right)\right\}, \\
p_{i 1}^{-}=\min \quad\left\{\left(a_{i} \cos ^{2} \theta_{M}+c_{i}\right),\left(a_{i} \cos ^{2} \theta_{M}+c_{i} \cos \theta_{M}\right),\right. \\
\left.\left(a_{i}+c_{i} \cos \theta_{M}\right),\left(a_{i}+c_{i}\right)\right\},
\end{gathered}
$$

then for $\forall \theta \in\left[-\theta_{M}, \theta_{M}\right]$ and $i=0,1,2$

$$
p_{i 1}^{-} \leq\left(a_{i} \cos ^{2} \theta+c_{i} \cos \theta\right) \leq p_{i 1}^{+} \text {. }
$$

Consider the two terms involving $\sin \theta$ (i.e., $b_{i} \cos \theta \sin \theta+d_{i} \sin \theta$ ) in $\underline{(16)}$. If we denote 


$$
\begin{aligned}
& p_{i 2}^{+}=\max \left\{0,\left(b_{i}+d_{i}\right) \sin \theta_{M}\left(b_{i} \cos \theta_{M}+d_{i}\right) \sin \theta_{M}\right\}, \\
& p_{i 2}^{-}=\min \left\{0,\left(b_{i}+d_{i}\right) \sin \theta_{M},\left(b_{i} \cos \theta_{M}+d_{i}\right) \sin \theta_{M}\right\} .
\end{aligned}
$$

Then, for $\forall \theta \in\left[-\theta_{M}, \theta_{M}\right]$ and $i=0,1,2$

$$
p_{i 2}^{-} \leq\left(b_{i} \cos \theta \sin \theta+d_{i} \sin \theta\right) \leq p_{i 2}^{+} \text {(20) }
$$

Thus,

$$
\left(p_{i 1}^{-}+p_{i 2}^{-}+e_{i}\right) \leq q_{i} \leq\left(p_{i 1}^{+}+p_{i 2}^{+}+e_{i}\right) \cdot(21)
$$

If we denote:

$$
\begin{aligned}
& q_{M i}=p_{i 1}^{+}+p_{i 2}^{+}+e_{i}, \\
& q_{m i}=p_{i 1}^{-}+p_{i 2}^{-}+e_{i},
\end{aligned}
$$

then, the bounds for $q_{i}$ 's are determined:

$$
q_{m i} \leq q_{i} \leq q_{M i}, i=0,1,2,(24)
$$

where all $q_{m i}$ 's and $q_{M i}$ 's are functions of the admittance matrix (independent of the configuration).

\section{A.2 Sufficient Conditions for Error-Reduction}

Since the bounds of $q_{i}{ }^{\prime} s$ are determined, a single-variable polynomial is constructed for which the method used for single-point contact case ${ }^{[12]}$ is applied.

First, the error-reduction condition must be satisfied at one configuration in the range considered (say, at $[\theta, \delta]=[0,0]$ ).

To consider all configurations, we construct a polynomial by:

$$
F_{p}(\delta)=q_{M 2} \delta^{2}+q_{M 1} \delta+q_{m 0}
$$

where the coefficients are constants defined in (22) and (23). Denote

$$
q_{M}=\max \left\{\left|q_{M 2}\right|,\left|q_{M 1}\right|\right\},(26)
$$

It is proved ${ }^{[12]}$ that, if

$$
\frac{\left|q_{m 0}\right|}{q_{M}+\left|q_{m 0}\right|}>\delta_{M},(27)
$$


then, $F_{p}(\delta)$ has no root in $\left[-\delta_{M}, \delta_{M}\right]$. Since the coefficients of $F_{p}(\delta)$ are extremal values of $q_{i}$ 's in the range considered, the condition (27) ensures that the function $F_{e r}(\theta, \delta)$ in $\underline{(15)}$ has no root in $\left[-\delta_{M}, \delta_{M}\right]$ for any given $\theta \in\left[-\theta_{M}, \theta_{M}\right]$. In fact, for a given $\theta \in\left[-\theta_{M}, \theta_{M}\right], F_{e r}(\theta, \delta)$ is a polynomial in $\delta$. As shown in [12], a root of $F_{e r}(\theta, \delta), \delta_{\theta}$, must satisfy which ensures that $F_{\mathrm{er}}\left(\theta^{\prime}, \delta\right)$ has no root in $\left[-\delta_{M}, \delta_{M}\right]$. Therefore, constraints $\underline{(13)}$ and $\underline{(27)}$ are a set of sufficient conditions for error-reduction for all configurations in a contact state.

$$
\delta_{\theta} \geq \max \left\{\frac{\left|q_{0}\right|}{\left|q_{1}\right|+\left|q_{0}\right|}, \frac{\left|q_{0}\right|}{\left|q_{2}\right|+\left|q_{0}\right|}\right\} \geq \frac{\left|q_{\mathrm{m} 0}\right|}{q_{M}+\left|q_{m 0}\right|}>\delta_{M}
$$

\section{B. Conditions for $\{e-v, e-v\}$ Contact State}

In this case, the two-point contact wrench is a combination of the two $\{e-v\}$ contact wrenches.

Using the notation developed in Section III-B, the contact wrenches for the two $\{e-v\}$ contacts are obtained by (11):

$$
\mathbf{w}_{i}=\left[\begin{array}{c}
\mathbf{n}_{i} \\
{\left[\left(\mathbf{r}_{c i}+\mathbf{r}_{e i} \delta_{i}\right) \times \mathrm{n}_{\mathrm{i}}\right] \cdot \mathrm{k}}
\end{array}\right], i=1,2(28)
$$

where $\mathbf{r}_{c i}$ and $\mathbf{r}_{e i}$ are constant vectors associated with edge $i$.

By (8), the error reduction function can be expressed in terms of two variables $\delta_{1}$ and $\delta_{2}$ :

$$
\begin{gathered}
F\left(\delta_{1}, \delta_{2}\right)=\left(a_{2} \delta_{1}^{2}+b_{2} \delta_{1}+c_{2}\right) \delta_{2}^{2}+\left(a_{1} \delta_{1}^{2}+b_{1} \delta_{1}+c_{1}\right) \delta_{2} \\
+\left(a_{0} \delta_{1}^{2}+b_{0} \delta_{1}+c_{0}\right)
\end{gathered}
$$

where $a_{i}{ }^{\prime} \mathrm{S}, b_{i}{ }^{\prime} \mathrm{S}$ and $c_{i}{ }^{\prime} \mathrm{S}$ are all functions of the admittance matrix $\mathbf{A}$.

Denote

$$
q_{i}\left(\delta_{1}\right)=a_{i} \delta_{1}^{2}+b_{i} \delta_{1}+c_{i}, i=0,1,2 .
$$

Since the $q_{i}{ }^{\prime}$ S are quadratic functions, it is not difficult to determine their extreme values for $\delta_{1} \in\left[-\delta_{1 M}, \delta_{1 M}\right]$. In fact, if we denote

$$
\begin{gathered}
q_{m i}=\min \left\{q_{i}\left(-\delta_{1 M}\right), q_{i}\left(\delta_{1 M}\right), q_{i}\left(\frac{b_{i}}{2 a_{i}}\right)\right\}, \\
q_{M i}=\max \left\{q_{i}\left(-\delta_{1 M}\right), q_{i}\left(\delta_{1 M}\right), q_{i}\left(\frac{b_{i}}{2 a_{i}}\right)\right\}, \\
q_{M}=\max \left\{q_{M 1}, q_{M 2}\right\},
\end{gathered}
$$

then, for all $\delta_{1} \in\left[-\delta_{1 M}, \delta_{1 M}\right]$. 


$$
q_{m i} \leq q_{i} \leq q_{M i} \cdot(34)
$$

By the same reasoning used for the $\{e-v, v-e\}$ contact case in Section V-A2, a similar condition is obtained

$$
\frac{\left|q_{m 0}\right|}{q_{M}+\left|q_{m 0}\right|}>\delta_{M}
$$

This condition ensures that $F_{e r}\left(\delta_{1}, \delta_{2}\right)$ has no root for all $\delta_{1} \in\left[-\delta_{1 M}, \delta_{1 M}\right]$ and $\delta_{2} \in\left[-\delta_{2 M}, \delta_{2 M}\right]$. Therefore, constraints (13) and (35) are a set of sufficient conditions for error-reduction for all configurations in a contact state.

\section{Conditions for $\{v-e, v-e\}$ Contact State}

In this case, the two-point contact wrench is a combination of the two $\{v-e\}$ contact wrenches.

Using the notation developed in Section III-B, the contact wrenches for the two $\{v-e\}$ contact are obtained by (9):

$$
\mathbf{w}_{i}=\left[\begin{array}{c}
\mathbf{R} \mathbf{n}_{i} \\
\left(\mathbf{r}_{i} \times \mathbf{R n}_{i}\right) \cdot \mathbf{k}
\end{array}\right], i=1,2
$$

The error-reduction function (8) can be used directly. Since the wrenches involve only one variable $\theta$, the error-reduction function is a single-variable function in the form:

$$
\begin{aligned}
F_{e r}(\theta)= & a_{1} \cos ^{4} \theta+a_{2} \cos ^{3} \theta \sin \theta+a_{3} \cos ^{2} \theta \sin \theta \\
& +a_{4} \cos \theta \sin \theta+a_{5} \cos \theta+a_{6} \sin \theta+a_{0},
\end{aligned}
$$

where $a_{i}$ 's are functions of the admittance matrix A.

If we denote

$$
\begin{aligned}
& p_{1}(\theta)=a_{1} \cos ^{4} \theta+a_{5} \cos \theta+a_{0}, \\
& p_{2}(\theta)=a_{2} \cos ^{3} \theta+a_{3} \cos ^{2} \theta+a_{4} \cos \theta+a_{6},
\end{aligned}
$$

then $F_{e r}(\theta)$ can be expressed as:

$$
F_{\text {er }},(\theta)=p_{1}(\theta)+p_{2}(\theta) \sin \theta
$$

Since $|\theta| \leq \theta_{M} \ll \frac{\pi}{4}$, the bounds for the single-variable functions $p_{1}(\theta)$ and $p_{2}(\theta)$ can be obtained by the approach used for the single-point contact case ${ }^{[12]}$.

Let $p_{m i}$ and $p_{M i}$ be the bounds of $p_{i}$, i.e., 


$$
\begin{aligned}
& p_{m 1} \leq p_{1} \leq p_{M 1} \\
& p_{m 2} \leq p_{2} \leq p_{M 2}
\end{aligned}
$$

and

$$
p_{M}=\max \left\{\left|p_{2 m}\right|,\left|p_{2 M}\right|\right\} .
$$

Then, it can be proved that, the conditions

$$
\begin{aligned}
& p_{1 m}-p_{M} \sin \theta_{M}<0 \\
& p_{1 m}+p_{M} \sin \theta_{M}<0
\end{aligned}
$$

ensure that for all $\theta \in\left[-\theta_{M}, \theta_{M}\right], F_{e r}(\theta)<0$. Therefore, constraints (44)-(45) combined with (13) are a set of sufficient conditions for error-reduction for all configurations in a contact state.

\section{SECTION V.}

\section{Summary}

In this paper, we identified procedures for selecting the appropriate admittance to achieve reliable planar force-guided. assembly for multi-point contact cases. Conditions imposed on the admittance matrix for each of the various types of two-point contact are presented.

We show that, for bounded misalignments, if the conditions are satisfied for a finite number of contact configurations, the conditions ensure that force guidance is achieved for all configurations within the specified bounds.

In this study, two variables are used to describe the contact wrenches for a rigid body (with 1DOF) in two-point contact. With this approach, the error-reduction function is expressed in two independent variables regardless of the geometry of the contact. For each type of contact, by evaluating the bounds on the coefficients in the error-reduction function, specific conditions for satisfying error-reduction are obtained.

\section{ACKNOWLEDGMENT}

This research was supported in part by a Ford Motor Company URP award, and the National Science Foundation under grant IIS 0010017.

\section{References}

1. D. E. Whitney, "Force feedback control of manipulator fine motions," ASME Journal of Dynamic Systems, Measurements and Control, vol. 98, no. 2, 1977.

2. D. E. Whitney, "Quasi-static assembly of compliantly supported rigid parts," ASME Journal of Dynamic Systems, Measurements, and Control, vol. 104, no. 1, pp. 65-77, 1982. 
3. M. A. Peshkin, "Programmed compliance for error-corrective manipulation," IEEE Transactions on Robotics and Automation, vol. 6, no. 4, pp. 473-482, 1990.

4. H. Asada, "Teaching and learning of compliance using neural net," IEEE Transactions on Robotics and Automation, vol. 9, no. 6, pp. 863-867, 1993.

5. E. D. Fasse and J. F. Broenink, "A spatial impedance controller for robotic manipulation," IEEE Transactions on Robotics and Automation, vol. 13, no. 4, pp. 546-556, 1997.

6. Jr. Marcelo H. Ang and Gerry B. Andeen, "Specifying and achieving passive compliance based on manipulator structure," IEEE Transactions on Robotics and Automation, vol. 11, no. 4, pp. 504-515, 1995.

7. J. M. Schimmels and M.A. Peshkin, "Admittance matrix design for force guided assembly," IEEE Transactions on Robotics and Automation, vol. 8, no. 2, pp. 213-227, 1992.

8. J. M. Schimmels and M. A. Peshkin, "Force-assembly with friction," IEEE Transactions on Robotics and Automation, vol. 10, no. 4, pp. 465-479, 1994.

9. J. M. Schimmels, "A linear space of admittance control laws that guarantees force-assembly with friction," IEEE Transactions on Robotics and Automation, vol. 13, no. 5, pp. 656667, 1997.

10. J. M. R. Martinez and J. Duffy, "On the metric of rigid body displacements for infinite and finite bodies," ASME Journal of Mechanical Design, vol. 117, no. 1, pp. 41-47, 1995.

11. S. Huang and J. M. Schimmels, "Sufficient conditions used in admittance selection for planar force-guided assembly," in Proceedings of the IEEE International Conference on Robotics and Automation, Washington, D.C., May 2002, pp. 538-543.

12. S. Huang and J. M. Schimmels, "Sufficient conditions used in admittance selection for force-guided assembly of polygonal parts," IEEE Transactions on Robotics and Automation, (submitted for publication).

13. M. S. Ohwovoriole and B. Roth, "An extension of screw theory," ASME Journal of Mechanical Design, vol. 103, no. 4, pp. 725-735, 1981. 